\title{
Die Aufgabe der Endlichkeit
}

\author{
Eine theologische Replik auf Hartmut Rosas Theorie \\ sozialer Beschleunigung
}

Friederike Rass

Immer wenn ich dich besuch, fühl ich mich
grenzenlos. / Alles andere ist von hier aus
so weit weg. / Ich mag die Ruhe hier
zwischen all den Bäumen, / als ob es
den Frieden auf Erden wirklich gibt
Andreas Frege

Die Erfahrung einer vornehmlich säkular geprägten Moderne, radikal auf die eigene Endlichkeit zurückgeworfen zu werden, führt nach der Theorie des Soziologen Hartmut Rosa zu einer »sozialen Beschleunigung«: Mit diesem Entwurf geht Rosa der Frage nach, weswegen trotz immenser technischer Erleichterungen der Moderne, die Prozesse wesentlich zu verkürzen in der Lage sind, dennoch ein Lebensgefühl der Zeitnot entsteht. Rosa strebt in seinem Entwurf dabei keine Auseinandersetzung mit einer theologischen Perspektive an. Er spricht die Relevanz einer tiefergehenden Auseinandersetzung mit den religiösen Hintergründen dieses gesellschaftlichen und kulturellen Wandels zwar verschiedentlich an, stellt eine solche Untersuchung in seinem Entwurf jedoch zurück. Auch wenn Rosa an einer entscheidenden Stelle seines Entwurfs also eine Parallele zu einer religiösen Weltsicht zieht, worauf gleich einzugehen sein wird, spielt dieser Aspekt für seinen Entwurf insgesamt nur eine sehr untergeordnete Rolle. Ihm gelingt auch ohne diesen Bezug eine äußerst scharfsinnige Analyse, wie die einzelnen charakteristischen Phänomene einer modernen Gesellschaftsordnung unter dem Aspekt der sozialen Beschleunigung zu systematisieren und zueinander in Beziehung zu setzen sind.

Dabei entzieht sich Rosa jedoch der Frage, wie der von ihm festgestellten Gesellschaftsstruktur der Moderne auf inhaltlicher Ebene $\mathrm{zu}$ begegnen ist. Es ist dieser Punkt, an dem eine tiefergehende Berücksichtigung eines religiösen, insbesondere christlichen Deutungshorizontes für das Dilemma des modernen Subjekts einen weiterführenden Beitrag leisten kann. 
Die Wirkmächtigkeit dieser Auseinandersetzungen um die Frage der Endlichkeit basiert dabei insbesondere auf der Durchschlagskraft des unmittelbaren individuellen Erfahrungshorizonts des Subjekts, das sich mit Erfahrungen der Endlichkeit konfrontiert sieht. Darin durchtrennt es die disziplinären Grenzen theoretischer Auseinandersetzung und eröffnet einen Denk-Raum, der dem existenziellen Gewicht dieses Phänomens allererst Rechenschaft trägt. Eine theoretische Auseinandersetzung mit dem Phänomen der Endlichkeit, die dessen lebensweltliche Relevanz nicht berücksichtigt, verfehlt somit ihren Sinn. Zugleich verweist die hohe Prägungskraft, die der Endlichkeit unserer Existenz für die Bewertung dieser zukommt, auf eine wesentliche Tiefendimension menschlicher Existenz.

Im Folgenden sollen so ausgehend von der Auseinandersetzung mit Rosas Entwurf einer sozialen Beschleunigung zwei Dimensionen von Endlichkeit unterschieden und näher betrachtet werden. Zunächst wird unter Berücksichtigung der besonderen Bedingungen der Gegenwart auf die Frage eingegangen, welche Veränderungen unser Endlichkeitsbewusstsein in einem hoch technisierten und zunehmend säkularen Zeitalter erfährt und welche Konsequenzen dies für das Selbstverständnis des Subjekts der Gegenwart mit sich bringen kann. Darauf aufbauend soll ein alternatives Deutungsmuster für eine mögliche Neukontextualisierung des erarbeiteten, spätmodernen Endlichkeitsverständnisses aufgezeigt werden. Ziel ist die sachbezogene Überwindung disziplinärer Grenzen, die nicht nur auf deren ursprüngliche Bezogenheit verweist, sondern deren Potenzial für nicht weniger als die Frage nach dem Sinn von Sein neu erschließt.

\section{Der Kreislauf der Beschleunigung}

Hartmut Rosa legt in seinem Werk Beschleunigung. Die Veränderung der Zeitstrukturen der Moderne (2005) einen eindrücklichen Versuch vor, die strukturellen Entwicklungen der Gegenwart aus zeitsoziologischer Sicht auf ihre Wechselwirkungen mit der individuellen Ebene hin zu befragen. Ausgangspunkt seiner Überlegungen bildet dabei das Paradoxon, welches für ihn die moderne, durch technische Errungenschaften geprägte Gesellschaft wesentlich charakterisiert: "Wir haben keine Zeit, obwohl wir sie im Überfluss gewinnen. ${ }^{1}$ Hiervon ausgehend untersucht Rosa die Frage, inwiefern dieses Empfinden

1 Hartmut Rosa, Beschleunigung. Die Veränderung der Zeitstrukturen der Moderne, Frankfurt a.M. 2005, 11. 
von Zeitknappheit in der Moderne wissenschaftlich erfasst und bewertet werden kann. Die entscheidende Grundlage seiner Überlegungen bildet dabei seine Definition von Beschleunigung.

Diese entwickelt Rosa nicht allein ausgehend vom Faktor Zeit, sondern berücksichtigt dessen Korrelation mit dem Faktor »Menge«. Was abstrakt scheint, wird im Beispiel rasch plausibel: So geht es nicht allein darum, dass in der Moderne unsere Kommunikation durch eine technisch ermöglichte Verkürzung unserer Kommunikationswege enorm beschleunigt wird. Erst in Korrelation mit der Menge der tatsächlich vorgenommenen Kommunikationsvorgänge lässt sich der Eindruck einer Zeitverknappung in der Moderne plausibel machen. So verkürzt die Einführung des Emailsystems einen einzelnen Kommunikationsvorgang zwar deutlich, die Menge der darüber vorgenommenen Kommunikationsvorgänge übersteigt jedoch zugleich jede frühere mittelbare Kommunikationsform auf überproportionale Weise. Die dadurch vermeintlich erreichte "Zeitersparnis« wird aus diesem Grund nicht nur zunichte gemacht, faktisch steigt der dieser Kommunikationsform gewidmete Zeitaufwand vielmehr. ${ }^{2}$

Es kommt zum paradoxen Geschehen, dass entgegen der technisch ermöglichten Verkürzung der Zeit, die für wesentliche alltagsbestimmende Vorgänge wie Kommunikation, Transportwege oder auch Haushaltsaufgaben aufgewendet werden muss, im Resultat tatsächlich mehr Zeit für diese Bereiche aufgewendet wird. Ein Verständnis der gesellschaftlichen Verfasstheit der Moderne als "Beschleunigungsgesellschaft « ${ }^{3}$ hängt für Rosa damit sowohl vom Faktor "Beschleunigung" wie auch vom Faktor »Wachstum"ab. Relevant ist, dass beide Faktoren dabei keineswegs kausal miteinander verbunden sind: Eine Steigerung der Beschleunigungsrate bedingt nicht notwendig eine Steigerung der Wachstumsrate. ${ }^{4}$ Oder, um im Beispiel zu bleiben: Nur weil die Email ein rascheres Versenden von Nachrichten technisch ermöglicht, müsste dies nicht zwangsläu-

\footnotetext{
2 Vgl. zu diesem Beispiel sowie den zu Grunde liegenden Berechnungen Rosa, Beschleunigung, 117-120.

3 Ebd., 120.

4 Vgl. hierzu auch Rosas Verweis auf Niklas Luhmann, Die Knappheit der Zeit und die Vordringlichkeit des Befristeten, in: Ders., Politische Planung. Aufsätze zur Soziologie von Politik und Verwaltung, Wiesbaden ${ }^{4} 1994,143-164$, 156: Luhmann konstatiert bereits 1968 die systemrelevante Funktion eines steigenden Geschwindigkeitsdrucks, der "umso merkwürdiger [ist], als unser Zeitbegriff ins Unendliche geht und uns keinerlei letzten Termin, kein Weltende suggeriert. Wir hätten demnach Zeit, unendlich viel Zeit.»
} 
fig eine Erhöhung der Menge an Nachrichten nach sich ziehen. ${ }^{5}$ Entsprechend zieht Rosa die Konsequenz: »Die [...] Zeitknappheit der Moderne, entsteht nicht weil, sondern obwohl auf nahezu allen Gebieten des sozialen Lebens enorme Zeitgewinne durch Beschleunigung verzeichnet werden. $\ll^{6}$

Zeit wird gerade trotz der Privilegien technischer Neuerungen somit auf individueller Ebene als »knapp« empfunden. Das zweite Paradoxon im Hinblick auf seine Feststellung einer umfassenden "Zeitnot und Beschleunigungssehnsucht" ${ }^{7}$ der Gegenwart stellt für Rosa der Wegfall einer Endzeiterwartung in der Moderne dar. Dabei setzt er ein modernes Zeitverständnis voraus, das er als »linear mit offener Zukunft» definiert, also als "Vorstellung einer entwicklungsoffenen [...] sich linear aus der Vergangenheit herausentwickelnden Zukunft unserer Zeiterfahrung ${ }^{8}$. So lässt sich seines Erachtens für die Gesellschaft der Gegenwart keine Erwartung eines apokalyptischen Weltendes heranziehen, welches den Eindruck genereller Zeitnot rechtfertigen würde. Zugleich entbehrt die Idee einer »kategorial neuen, besseren Zukunft " ${ }^{9}$, die erstrebenswert und aus diesem Grund auf irgend eine Weise rascher herbeizuführen wäre, so jeder Grundlage. Wie also lässt sich das Phänomen einer Gesellschaftsform begründen, die eine kontinuierlich zunehmende soziale Beschleunigung erfährt und diese zugleich von sich verlangt? ${ }^{10}$

Rosa grenzt sich in seinem Antwortversuch wesentlich von sozialwissenschaftlichen Positionen ab, die die Beschleunigungstendenz der Moderne auf eine rein ökonomische Grundlegung zurückführen, um analoge kulturelle sowie soziale Strukturen dann als schlichte

5 Vgl. Rosa, Beschleunigung, 119f., Fn. 11.

6 Ebd., 117 (Hervorhebung F.R.).

7 Ebd., 281.

8 Ebd.

9 Ebd.

${ }^{10}$ Ein eindrückliches aktuelles Beispiel hierfür bildet die Reaktion auf die diesjährigen weihnachtlichen Quartalszahlen des Online-Versandhandels Amazon: „Der weltgrößte Online-Einzelhändler Amazon hat im vierten Quartal seinen bislang größten Gewinn gemacht - die Erwartungen der Anleger aber dennoch enttäuscht. Von Oktober bis Dezember stieg der Überschuss um mehr als die Hälfte verglichen mit dem Vorjahreswert auf 482 Millionen Dollar (440 Mio. Euro), wie der Konzern mitteilte. Das Weihnachtsgeschäft spülte Amazon so viel Geld in die Kasse wie noch nie zuvor in der 19-jährigen Börsengeschichte des Unternehmens. Doch an der Wall Street kam der Geschäftsbericht trotzdem schlecht an - die Amazon-Aktie stürzte nachbörslich zeitweise um mehr als zwölf Prozent ab. Analysten hatten mit einem deutlich höheren Gewinn und auch mit mehr Umsatz gerechnet. Die Erlöse legten im Schlussquartal um 22 Prozent zum Vorjahr auf 35,7 Milliarden Dollar zu. «ttps:// www.tagesschau.de/wirtschaft/amazon-177.html (31.01.2016). 
Folgephänomene einzuordnen. ${ }^{11}$ Eine rein ökonomische Begründungsstruktur ist seines Erachtens insbesondere nicht in der Lage, die »ideellen und kulturellen Voraussetzungen dafür, dass die Produktiv- und Beschleunigungskräfte auf die beschriebene Weise entfesselt werden konnten ${ }^{12}$, zu erfassen. Dies führt seines Erachtens zu einer Simplifizierung des erklärungsbedürftigen Phänomens, zu dessen Veranschaulichung er so verschiedene Konkretisierungen wie Fast Food, Multitasking oder auch Speed Dating anführt. Im Gegenzug plädiert er vielmehr für einen zirkulären Prozess der wechselseitigen Bedingung der verschiedenen Faktoren, wobei er eine technische Beschleunigung (1.), die Beschleunigung des sozialen Wandels (2.) sowie die Beschleunigung des individuellen Lebenstempos (3.) voneinander unterscheidet. ${ }^{13}$ Gegen ein ökonomisches beziehungsweise ökonomisiertes Deutungsschema argumentiert er damit für ein multifaktorielles Ursachengefüge, das sich wechselseitig bedingt und nicht auf einen seiner Aspekte reduziert werden kann.

Für eine Näherbestimmung des Phänomens der Endlichkeit ist vor diesem Hintergrund nun insbesondere Rosas Verhältnisbestimmung des sozialen Wandels der Gegenwart zum individuellen Lebenstempo entscheidend. Im Blick hierauf konstatiert er eine „Verkürzung der Stabilität von Zeithorizonten«, was dazu führt, dass "Handlungsbedingungen und Situationsbestimmungen $[\ldots]$ ihre Geltungskonstanz in immer kürzeren Zeitabständen " $^{14}$ verlieren. Die Deutungshorizonte der Gegenwart überlagern sich zunehmend und lösen sich zugleich in immer rascherer Folge wechselseitig ab. Im Zuge dessen verringern sich auf der Ebene des Individuums konsequent die Kapazitäten, die immer rascheren gesellschaftlichen Entwicklungen der Gegenwart zu erfassen und darauf zu reagieren. Die Zeit wird auf subjektiver Ebene tatsächlich knapp: Die Erfahrung der Endlichkeit des Individuums (3.) steht in immer stärkerem Kontrast zur sich scheinbar unendlich steigernden Entwicklung seiner Umwelt (2.). Um dieser Anforderung gerecht werden zu können, treten Forderungen nach technischen Lösungen (1.) auf, die helfen, dieser Beschleunigung wieder Herr werden zu können. Diese Möglichkeiten führen dann wiederum zur Beschleunigung des sozialen Wandels (2.), was wiederum erneut nach einer Beschleunigung des individuellen Lebenstempos (3.) ruft: Ein sich selbst tragender

\footnotetext{
${ }^{11}$ Vgl. Rosa, Beschleunigung, 279.

12 Ebd., 280.

${ }^{13} \mathrm{Vgl}$. hierzu sowie für den folgenden Abschnitt insbesondere Rosa, Beschleunigung, $243 \mathrm{ff}$.

${ }^{14}$ Ebd., 248.
} 
Beschleunigungszirkel ist entstanden, der für Rosa die moderne Gesellschaft charakterisiert und nur schwer zu durchbrechen erscheint.

\section{Die Revolution frisst ihre Kinder?}

Spannend wird dieses von Rosa aus soziologischer Perspektive beschriebene Dilemma des neuzeitlichen Subjekts, setzt man es zu einer theologischen Perspektive in Beziehung. Rosa selbst macht eine Andeutung in dieser Richtung, wenn er konstatiert:

"Das eigentliche, verborgene, aber kulturell höchst wirksame 'Heilsversprechen der sozialen Beschleunigung [...] besteht darin, dass sie ein säkulares funktionales Äquivalent für die Idee des sewigen Lebens zu bieten scheint und daher als die Antwort der Moderne auf das unvermeidliche große Kulturproblem der menschlichen Endlichkeit, den Tod, verstanden werden kann. $\otimes^{15}$

An die Stelle einer religiösen Jenseitserwartung tritt für Rosa das Phänomen der sozialen Beschleunigung: Das Subjekt wird auf sich selbst zurückgeworfen und kompensiert diese Erfahrung der eigenen Endlichkeit mit einem Streben nach "mehr", das qua Definition nie erfüllt werden kann. Die traditionelle Funktion des "Jenseits«, das dem Tod eine Dimension der Hoffnung entgegenstellen konnte und nach Rosa somit dessen Endgültigkeit zu entkräften in der Lage war, wird für ihn so von einer modernen Beschleunigungssucht abgelöst.

Für Rosa selbst verbleibt die Funktion von Religion für diesen Ablösungsprozess ambivalent. So erwähnt er auf der einen Seite mit Verweis auf Ernst Benz den möglichen Einfluss einer christlichen Jenseitserwartung auf eine beschleunigte Wahrnehmung der Jetzt-Zeit. ${ }^{16}$ Auf der anderen Seite würdigt er zugleich die stabilisierende Wirkung, die einem transzendenten Zeitverständnis des Christentums zukommen kann. Diese Spannung löst Rosa für sich, indem er der christlichen Jenseitserwartung zunächst eine Beschleunigungsdynamik zuschreibt, die dann jedoch "mit jenen religiösen Vorstellungen [einer jenseitigen Heilserwartung] unvereinbar wurde und sie daher zu erodieren begann ${ }^{17}$. So kann seines Erachtens aus der christlichen Jenseitsvorstellung zunächst ein Drang erwachsen sein, die Ankunft des Erhofften zu beschleunigen. Die dadurch angestoßenen Entwicklungen führen dann jedoch dazu, dass einer

${ }^{15}$ Ebd., 287.

${ }^{16}$ Ebd., 281.

17 Ebd., 287, Fn. 78. 
religiösen Weltdeutung keine Deutungsmacht mehr zugesprochen wird und sie sich so letztlich selbst überholt. ${ }^{18}$

Rosa verortet die soziale Beschleunigung der Gegenwart hier sehr spezifisch als Reaktion auf den Tod als »das unvermeidliche große Kulturproblem der menschlichen Endlichkeit«. Dennoch erfasst er die weit über diese Engführung hinausgehende Reichweite des damit verbundenen Problemkomplexes sehr genau: Auch wenn sich Rosa im Kontext dieses Zitats insbesondere auf die temporale Begrenzung des menschlichen Lebens bezieht, verweist er darin doch zugleich auf die Übertragung dieses Wissens auf eine existenzielle Endlichkeitsvorstellung des Menschseins an sich. Die Frage, welche Funktion die Endlichkeit des Menschen für dessen Selbstverständnis einnehmen kann, umfasst so weit mehr als die Frage nach dem Umgang mit seiner Sterblichkeit und erstreckt sich bis hin zu den alltäglichsten seiner Bezüge. Dies wird auch in der Pluralität der von ihm herangezogenen, sehr pointiert gewählten Beispiele deutlich (nicht zuletzt etwa, wenn er Speed Dating trocken als »[b]eschleunigte Serien-Sichtung möglicher Intimpartner ${ }^{19}$ charakterisiert).

In seiner Parallelisierung der sozialen Beschleunigung als das »funktionale Äquivalent für die Idee des sewigen Lebens« verfehlt Rosa jedoch zugleich ein wesentliches Element dieser Vorstellung im Horizont des christlichen Glaubens. Für den Soziologen lässt sich die soziale Beschleunigung mit der Vorstellung des »ewigen Lebens" gleichsetzen, da beide dieselbe gesellschaftliche Funktion einnehmen. Sie dienen dazu, die Anforderungen einer Gesellschaft an das Individuum zu bewältigen, in der »die maximale Auskostung von Weltoptionen und die optimale Entfaltung eigener Anlagen $[\ldots]$ zum Paradigma gelingenden Lebens geworden ist $\aleph^{20}$. Im ersten Moment scheint diese Parallelisierung auch sehr überzeugend zu sein. Betrachtet man sie jedoch genauer, lässt sich die christliche Vorstellung eines "ewigen Lebens" nicht so einfach auf diese Weise in die Gleichung einsetzen, sondern steht einer solchen Funktionalisierung vielmehr gerade wesentlich entgegen. Kompensiert die moderne Gesellschaft den "Tod als Optionenvernichter « ${ }^{21}$ nach Rosa zunehmend durch den Versuch, die Anzahl der wahrgenommenen Optionen im »Diesseits« zu erhöhen, bildet die Vorstellung

${ }^{18}$ Diese sehr allgemein gehaltene Argumentation Rosas hakt offensichtlich gleich an mehreren Stellen. Auf diese wird hier jedoch nicht ausführlich eingegangen werden, da sie für seine Argumentation keine nachhaltige Bedeutung einnehmen, was gleich noch deutlich werden wird.

${ }^{19}$ Rosa, Beschleunigung, 114.

${ }^{20}$ Ebd., 474.

${ }^{21}$ Ebd. 
eines "Jenseits" eine weit komplexere Vorstellungswelt ab. In deren Vordergrund steht ein Bewusstwerden der eigenen Endlichkeit, die gerade nicht als selbst zu behebendes Defizit verstanden wird, wobei die Sterblichkeit des Menschen nur einen Aspekt dieser Endlichkeit ausmacht. Rosa reduziert die Jenseitsvorstellung in seiner Parallelisierung hingegen auf eine bloße, vom Tod kurz unterbrochene chronologische Verlängerung des Lebens und seiner Verwirklichungsoptionen ins Unendliche.

Gemeinsam mit Rosa bleibt dabei der Fokus, dass der Mensch sich seiner eigenen Endlichkeit bewusst wird. Unmittelbar damit verbunden ist die Erfahrung, dass die Endlichkeit der Existenz aus eigener Kraft gerade nicht aufhebbar zu sein scheint. Soweit ließe sich Rosas These einer Äquivalenz der religiösen Jenseitsvorstellung $\mathrm{zu}$ seiner These einer sozialen Beschleunigung noch halten: Wo nach Rosa heute mittels sozialer Beschleunigung versucht wird, die Endlichkeitserfahrung im "Diesseits" zu kompensieren, steht für ihn im religiösen Kontext die Idee des »ewigen Lebens", die diese Kompensationsleistung auf ein Leben nach dem Tod verlegen kann. Entscheidend und wesentlich verschieden von Rosas funktionaler Gleichsetzung ist aus christlicher Perspektive dann jedoch der Sachverhalt, dass diese Erfahrung der eigenen Endlichkeit gerade keinen Mangel darstellen muss. So verweist sie auf einen alternativen Deutungshorizont, der gegenüber dem von Rosa festgestellten Kompensationsmechanismus eine kritische Funktion einnehmen kann.

\section{Anerkennung der Endlichkeit als Öffnung des Kreislaufs der Beschleunigung}

Der Theologe Fulbert Steffensky skizziert das von Rosa erfasste Dilemma des modernen Subjekts in einem Vortrag auf struktureller Ebene ganz ähnlich, kontextualisiert dieses jedoch im Denkhorizont des christlichen Glaubens:

"Wer an Gott glaubt, braucht nicht Gott zu sein und Gott zu spielen. Er muss nicht der Gesündeste, der Stärkste, der Schönste, der Erfolgreichste sein. Er ist nicht gezwungen, völliger Souverän seines eigenen Lebens zu sein. Wo aber der Glaube zerbricht, da ist dem Menschen die nicht zu tragende Last derVerantwortung für die Ganzheit auferlegt. $\mathbb{1 2}^{22}$

${ }^{22}$ Fulbert Steffensky, Der Schmerz und die Gnade der Endlichkeit, Vortrag, hg. v. Hospizarbeit im Ev. Johanneswerk e.V., Bielefeld 2006, 8. 
Die Diagnose lautet bei Steffensky wie bei Rosa auf funktionaler Ebene so zunächst gleich: In Abwesenheit eines transzendenten Glaubenshorizonts wird dem Subjekt der Gegenwart die alleinige Verantwortung für das Gelingen seiner Existenz auferlegt. Erschwerend kommt die exponentielle Zunahme der Realisierungsmöglichkeiten des eigenen Lebens sowie der Lebenskontexte in der Gegenwart hinzu. Rosa bleibt in seinem Befund einer sozialen Beschleunigung dann jedoch stehen, auch wenn er damit am Ende seiner Untersuchung die "Hoffnung [verbindet], dass in ihr bereits die Umrisse einer kritischen Gesellschaftstheorie sichtbar werden ${ }^{23}$. Auch wenn er im Zuge dessen verschiedene Szenarien andeutet, in welche Richtung sich seine Theorie hinsichtlich der Beschleunigungsdynamik der Gegenwart weiterdenken ließe, muss er bis auf wenige Hinweise letztlich jedoch offen lassen, wie sich deren kritisches Potenzial tatsächlich in die Gegenwart hinein auswirken könnte.

Anders als Rosa bezieht sich Steffensky jedoch nicht allein auf die soziokulturellen Bezüge des modernen Individuums, sondern verortet seine Überlegungen zusätzlich auf einer grundlegend existenziellen Ebene. Auch wenn deren Verortung im christlichen Denkhorizont in der modernen Gesellschaft eine zunehmende Entfremdung erfahren mag, besitzt sie dessen ungeachtet hohe Erklärkraft. Dies zeigt sich insbesondere an der Stelle, an der der soziologische Entwurf aufgeben muss. So entwickelt Steffensky die Frage, wie mit dem zunehmend empfundenen Druck der Selbstoptimierung umgegangen werden kann, an der der Mensch jedoch angesichts der sich ständig verändernden Deutungshorizonte dieses Prozesses zugleich notwendig scheitern muss, vor dem Horizont des christlichen Glaubens folgendermaßen: Die insbesondere im Tod präsente Erfahrung der eigenen Endlichkeit stellt nicht primär eine Bedrohung dar, sondern bildet vor allem anderen eine fundamentale Entlastung. Gelingt es, die im Tod kulminierende Erfahrung der temporalen Begrenztheit des eigenen Lebens auf die Vollzugsdimension des eigenen Lebens zu beziehen, resultiert daraus zweierlei.

Zum einen wird der illusionäre Charakter eines vollkommenen Lebens enttarnt und letzteres gänzlich neu bewertet. Die Endlichkeit wird als wesentlicher Teil gelungenen Lebens verstanden. Dessen "Vollkommenheit" ist somit gerade im Horizont dieser Endlichkeit zu suchen: "Das Leben ist endlich, nicht nur weil wir sterben müssen, Die Endlichkeit liegt im Leben selbst, im begrenzten Glück, im begrenzten Gelingen [...]. Wenn man [...] der Endlichkeit fähig wäre,

\footnotetext{
${ }^{23}$ Rosa, Beschleunigung, 480.
} 
dann brauchte die eigene Bedürftigkeit, Schwäche, vielleicht sogar die Todesnähe nicht in Chaosängste stürzen. ${ }^{24}$ Steffensky denkt diesen Gedanken hier bis zur letzten Konsequenz durch, indem er mit dem Motiv spielt, dass selbst unsere Fähigkeit zur Endlichkeit endlich ist: "Wenn man der Endlichkeit fähig wäre ..." So lässt sich Rosas Diagnose der Beschleunigungssucht des modernen Individuums auf- und ernstnehmen, das Bedürfnis der Selbstoptimierung wird nicht einfach bagatellisiert. Zugleich erhält es jedoch ein Vorzeichen, das zu einer befreienden Re-evaluation dieses Bedürfnisses einlädt. Steffensky selbst plädiert für ein Lob der "gelungenen Halbheit» im Gegenüber zum »Totalitätsterror « ${ }^{25}$.

Damit wirkt er zum anderen einem resignativen Diskurs entgegen: Die Anerkennung der konstitutiven Endlichkeit des Menschseins ist gerade nicht als Kapitulation vor einer Unzahl an Realisierungsmöglichkeiten und Deutungshorizonten zu verstehen. Vielmehr ermöglicht eine solche Anerkennung allererst die kritische Auseinandersetzung mit den Möglichkeitsräumen der eigenen Existenz, ohne sich von diesen her in seinem Handeln absolut bestimmen zu lassen.

So kann, mit Steffensky gesprochen, gerade aus der kritischen Reflexion der systematischen Überforderung des modernen Subjekts schließlich ein "Mut zum fragmentarischen Handeln $\ll^{26}$ entstehen.

Dieser Lösungsansatz ist dabei gerade nicht als Spezialdiskurs zu verstehen, dem nur für christlich sozialisierte Individuen Erklärkraft zukommt. Die Erfahrung der Endlichkeit ist vielmehr ein universales existenzielles Phänomen, das sich nicht nur im Horizont des Glaubens als Beziehungsgeschehen erweisen kann:

"Das, wovon wir eigentlich leben, können wir nicht herstellen: nicht die Liebe, nicht die Freundschaft, nicht die Vergebung, nicht die eigene Ganzheit und Unversehrtheit. Man kann sich nicht selbst beabsichtigen, ohne sich zu verfehlen [...]. Gnade heißt Befreiung von dem Zwang, sein eigener Hersteller zu sein. $\mathbb{1}^{27}$

Diese Perspektive steht strukturell allen offen und bildet einen argumentativen Ausgangspunkt, um der Explosion der Möglichkeitsräume der Gegenwart eine kritische Perspektive entgegenzusetzen. So lässt sich ein Denkweg eröffnen, diese einerseits in ihrer

${ }^{24}$ Steffensky, Der Schmerz und die Gnade der Endlichkeit, 8f.

${ }^{25}$ Ebd., 8 .

${ }^{26}$ Ebd., 9.

${ }^{27}$ Ebd., 6. 
Vielfalt positiv zu würdigen und als Erweiterung beziehungsweise Unterbrechung des gegenwärtigen Horizonts wahrnehmen zu können, ohne ihnen zugleich eine radikale Bestimmung des Subjekts zuzusprechen. Darüber hinaus wird so nicht nur eine neue Selbstbestimmung des Subjekts ermöglicht, sondern auch eine neue Wahrnehmung der Mit-Welt des einzelnen Subjekts. Indem das Subjekt in der Anerkennung seiner eigenen Endlichkeit zugleich seine notwendigeVerwiesenheit von anderen her und auf andere hin erfassen kann, kann auch die Dimension der Gemeinschaftlichkeit wieder einen neuen Stellenwert einnehmen. Und dies ist, nicht nur, aber insbesondere auch in Zeiten einer so genannten "Flüchtlingskrise unverzichtbar.

Die eigene Endlichkeit anzunehmen und diese zugleich gerade nicht als Einschränkung, sondern in ihrer Näherbestimmung der eigenen Identität als befreiendes Moment zu erkennen, das ein neues Handeln ermöglicht, ist somit keineswegs eine rein theoretische Denkspielerei, sondern bildet vielmehr eine der wesentlichen Aufgaben unserer Zeit.

- Dr. des. Friederike Rass hat Evangelische Theologie, Politikwissenschaften und Philosophie in Tübingen, Hamburg und Buenos Aires studiert und wurde 2015 mit einer religionsphilosophischen Arbeit an der Universität Zürich promoviert. Derzeit arbeitet sie als Assistentin für Ethik an der Universität Tübingen. 